\title{
NOTIZEN
}

\section{Verbesserungen bei der Berechnung von Donatorniveaus durch Einführung einer effektiven Störstellenladung}

\section{Albert Haug}

II. Institut für Theoretische Physik der Technischen Universität Berlin

(Z. Naturforsch. 25 a, 143-144 [1970] ; eingegangen am 5. Dezember 1969)

Die Berechnung von Donatorniveaus mittels des Modells der wasserstoffartigen Störstelle läßt sich erheblich verbessern, wenn man die Donatoren nicht als einwertige Ionen auffaßt, sondern ihnen eine geeignete effektive Ladung zuschreibt, die man aus den Verhältnissen bei freien Atomen ableiten kann.

Bei der Berechnung von Störstellenniveaus mittels des Modells der wasserstoffartigen Störstelle erhält man zwar für Germanium befriedigende Ergebnisse, für Silizium dagegen erhebliche Abweichungen von den experimentellen Werten ${ }^{1}$. Außerdem kann das Modell die Unterschiede in der Lage der Störstellenniveaus nicht erklären, die bei verschiedenartigen Donatoren der 5 . Spalte des periodischen Systems (bzw. verschiedenen Akzeptoren der 3. Spalte) auftreten. Offenbar liegt dies daran, daß in diesem Modell alle derartigen Donatoren in ihrer Wirkung auf ein äußeres Elektron als einfach geladene Ionen dargestellt werden, entsprechend dem Umstand, daß sie gegenüber den Atomen des Wirtsgitters in der nächsten Spalte des periodischen Systems stehen. Als Störstellenpotential hat man daher das Coulomb-Potential einer positiven Einheitsladung im Kristall, der durch seine Dielektrizitätskonstante $\varepsilon$ eingeht. Es fehlt nicht an Versuchen ${ }^{2-5}$, durch eine Abänderung dieses Potentials zu günstigeren Ergebnissen zu kommen. Einerseits wird dabei eine ortsabhängige Dielektrizitätskonstante eingeführt, andererseits werden Korrekturen des Potentials in der Umgebung der Störstelle vorgenommen. Während MülleR ${ }^{3}$ und SCHECHTER $^{5}$ nur eine Verbesserung des Grundzustandsniveaus von Si gelingt, erhalten CsAvINSZKY ${ }^{2}$ sowie MORITA und NARA ${ }^{4}$ auch Unterschiede in den Niveaus bei verschiedenen Donatoren. Jedoch sind die Verbesserungen quantitativ unzureichend und der Gang der Energiewerte mit der Art des Donators nicht in Úbereinstimmung mit der Erfahrung. Außerdem sind die relativ guten Ergebnisse der letztgenannten Arbeit ${ }^{4}$ zum Teil darauf zurückzuführen, daß in dem Verfahren ein Ausdruck offen bleibt, der als anpaßbarer Parameter verwendet wird. Alle genannten Arbeiten enthalten überdies die Vereinfachung, daß die wirkliche Struktur des Leitungs-

Sonderdruckanforderungen an Prof. Dr. A. HAUG, II. Institut für Theoretische Physik der Technischen Universität Berlin, D-1000 Berlin 10, Ernst-Reuter-Platz 7.

1 W. KoHN, Shallow Impurity States in Silicon and Germanium, Solid State Physics 5, 257 [1957].

2 P. Csavinszky, J. Phys. Chem. Solids 24, 1003 [1963].

3 K. Müller, Z. Naturforsch. 20 a, 1476 [1965]. bands von $\mathrm{Si}$ durch kugelförmige Energieflächen approximiert wird.

Im folgenden soll eine wesentlich einfachere Korrektur des Störstellenpotentials entwickelt werden, die aber - wie sich zeigen wird - erheblich wirkungsvoller ist. Die Beschreibung der Störstelle als einwertiges Ion ist zunächst auf ein Störatom zugeschnitten, das im periodischen System direkt neben dem Gitteratom liegt, also z. B. auf P als Donator in Si. Bei den übrigen Donatoren der 5. Spalte nimmt man stillschweigend an, daß die zusätzlichen Kernladungen in ihrer Wirkung auf ein äußeres Elektron durch die Elektronen der entsprechenden abgeschlossenen Schalen abgeschirmt werden. In Wirklichkeit ist jedoch diese Abschirmung nicht vollkommen, so daß die Störstelle als ein Ion mit der effektiven Ladungszahl $\bar{Z} \neq 1$ zu betrachten ist. Entsprechendes gilt aber auch für den Donator, der direkt neben dem Gitteratom liegt. Auch hier hat man es nicht mit einem freien Ion, sondern einer zusätzlichen Kernladung im Atomverband zu tun, wobei die Abschirmungsverhältnisse zu berücksichtigen sind. Gegenüber der bisherigen Formulierung ist also im HamiltonOperator des Störstellenproblems die effektive Ladung zu berücksichtigen. In der effektiven Masse-Darstellung (vgl. ${ }^{1}$ ), auf der das Modell der wasserstoffartigen Störstelle beruht, nimmt er dann folgende Form an:

$$
H=E(-i \nabla)-\bar{Z} e^{2} / \varepsilon r .
$$

Es kommt nun darauf an, die effektive Ladungszahl $\bar{Z}$ zu berechnen. Dazu gehen wir folgendermaßen vor: Der Wert $\bar{Z}=1$, der dem bisherigen Modell entspricht, setzt voraus, daß sich der Donator in seiner Wirkung auf ein äußeres Elektron gegenüber dem Gitteratom genau durch eine Kernladung unterscheidet. Dies würde nur gelten, wenn sowohl im Donator wie im Gitteratom die übrigen Elektronen die entsprechenden Kernladungen vollständig abschirmen würden. Da dies nicht der Fall ist, ist dieser Wert um die Differenz der effektiven Kernladungen $Z^{*}$ des Donators und des Gitteratoms zu korrigieren:

$$
\bar{Z}=1+Z_{\mathrm{D}}^{*}-Z_{\mathrm{G}}^{*} .
$$

Werte von $Z^{*}$, die den Atomen infolge der mangelnden Abschirmung zuzuschreiben sind, kennt man aus der Berechnung der Energie der freien Atome. Entsprechend der Einelektronnäherung, die man sowohl beim reinen Kristall wie auch beim Störstellenproblem verwendet, interessieren wir uns dabei für die Energie eines Elektrons der äußersten Schale („Leuchtelektron“). Nach einem Verfahren von SlateR ${ }^{6}$ läßt sich diese

4 A. Morita u. H. Nara, J. Phys. Soc. Japan 21 [1966], Suppl. S. 234.

5 D. Schechter, J. Phys. Soc. Japan 26, 8 [1969].

6 Vgl. P. Gombás, Theorie und Lösungsmethoden des Mehrteilchenproblems der Wellenmechanik, § 37, Verlag Birkhäuser, Basel 1950. 
Energie für ein beliebiges Atom in der Form der Grundzustandsenergie des Wasserstoffatoms darstellen, wenn man dem Atomkern eine effektive Ladung

$$
Z^{*}=(Z-\gamma) / n^{*}
$$

zuschreibt. Dabei ist $Z$ die wirkliche Kernladung, $\gamma$ ein Abschirmfaktor und $n^{*}$ eine der äußersten Schale des Atoms zugeordnete Zahl. Die Regeln zur Berechnung von $\gamma$ laufen in unserem Falle darauf hinaus, daß jedes Elektron der äußersten Schale (mit Ausnahme des Leuchtelektrons) 0,35 Kernladungseinheiten abschirmt, jedes der nächstinneren Schale 0,85 und jedes weitere 1,0. Den Nummern der äußersten Schale $n=1 ; 2 ; 3$; $4 ; 5 ; 6$ entsprechen der Reihe nach die Zahlen $n^{*}=1$; $2 ; 3 ; 3,7 ; 4,0 ; 4,2$. Damit erhält man für die interessierenden Atome:

$$
\begin{array}{llllll} 
& \text { Si } & \mathrm{Ge} & \mathrm{P} & \mathrm{As} & \mathrm{Sb} \\
Z^{*}: & 1,383 & 1,527 & 1,600 & 1,703 & 1,575 .
\end{array}
$$

Unter der Voraussetzung, daß sich an der effektiven Kernladung der Atome oder zumindest an deren Differenz im Kristall nichts Wesentliches ändert, erhält man für die effektive Ladung $\bar{Z}$ der Störstelle nach (2) :

$$
\begin{array}{lllll} 
& & \mathrm{P} & \mathrm{As} & \mathrm{Sb} \\
\text { in } \mathrm{Si}: & \bar{Z}= & 1,217 & 1,320 & 1,192 ; \\
\text { in Ge: } & \bar{Z}= & 1,073 & 1,176 & 1,048 .
\end{array}
$$

Mit diesen Werten von $\bar{Z}$ ist das Energiespektrum des Hamilton-Operators (1) zu berechnen, wobei wir uns hier für die Grundzustandsenergie interessieren, deren Betrag der Ionisierungsenergie gleich ist. Da die Lösung für $\bar{Z}=1$ dem bisherigen Modell entspricht und daher bereits vorliegt ${ }^{1}$, ist es nur notwendig zu untersuchen, wie die Energie von $\bar{Z}$ abhängt. Im Falle einfacher kugelförmiger Energieflächen nimmt (1) die Form

$$
H=-\hbar^{2} \Delta / 2 m^{*}-\bar{Z} e^{2} / \varepsilon r
$$

7 C. Kittel u. A. H. Mitchell, Phys. Rev. 96, 1488 [1954]. an und hat als Eigenwerte wasserstoffähnliche Niveaus, die bekanntlich mit $\bar{Z}^{2}$ gehen. Bei $\mathrm{Si}, \mathrm{Ge}$ hat man jedoch als Energieflächen im Leitungsband Rotationsellipsoide, so daß

$$
H=-\frac{\hbar^{2}}{2 m_{\mathrm{t}}}\left(\frac{\partial^{2}}{\partial x^{2}}+\frac{\partial^{2}}{\partial y^{2}}\right)-\frac{\hbar^{2}}{2 m_{1}} \frac{\partial^{2}}{\partial z^{2}}-\frac{\bar{Z} e^{2}}{\varepsilon r}
$$

wird. Da in diesem Falle das Problem nicht mehr exakt lösbar ist, bedient man sich der Variationsmethode ${ }^{7}$. Dabei zeigt sich, daß auch hier die Grundzustandsenergie mit $\bar{Z}^{2}$ geht. Unter Verwendung der Werte für $\bar{Z}=1$ ergibt sich dann für die Ionisierungsenergie (in $\mathrm{meV}$ ) :

\begin{tabular}{c|c|c|c|c|c|c}
\multirow{2}{*}{ Donator } & \multicolumn{3}{|c|}{ Si } & \multicolumn{3}{c}{ Ge } \\
\cline { 3 - 7 } & $\bar{Z}=1$ & $\bar{Z}$ nach (5) & exp. & $\bar{Z}=1$ & $\bar{Z}$ nach (5) & exp. \\
\hline $\mathrm{P}$ & & 42,9 & 45,3 & & 10,6 & 12,4 \\
\hline As & \multirow{2}{*}{29} & 50,5 & 53,5 & 9,2 & 12,7 & 13,7 \\
\hline Sb & & 41,2 & 42,5 & & 10,1 & 9,7
\end{tabular}

Gegenüber dem bisherigen Modell der Störstelle als einwertiges Ion $(\bar{Z}=1)$ erhält man also durch die Berücksichtigung der effektiven Ladung $\bar{Z}$ durchwegs Verbesserungen, wie der Vergleich mit den experimentellen Werten zeigt. Diese Verbesserungen sind vor allem bei $\mathrm{Si}$ ganz erheblich. Insbesondere ergibt sich eine Deutung der Unterschiede bei verschiedenen Donatoren, die sowohl dem Gang nach als auch quantitativ in befriedigender Übereinstimmung mit dem Experiment ist. Abgesehen davon, daß die hier geschilderte Methode viel einfacher als alle eingangs genannten Verfahren ist, sind die Ergebnisse auch viel besser als dort bzw. bedürfen keines anpaßbaren Parameters wie in ${ }^{4}$.

Der Verfasser hat Herrn A. Tinnappel für einige vorläufige Berechnungen zu diesem Problem zu danken, die er im Rahmen einer Diplomarbeit durchgeführt hat. 